\title{
La radio escolar digital y su aporte al aprendizaje en la asignatura de Lenguaje y Comunicación en el Colegio Altazor
}

\section{The digital school radio and its contribution to learning in the subject of Language and Communication in the Altazor School.}

\author{
María Gricelda Catalán Abarca \\ Pontificia Universidad Católica de Chile \\ mariagriceldacatalan@gmail.com
}

Resumen En el marco del logro de competencias comunicacionales y la necesidad de desarrollar habilidades acorde a los contextos socio-culturales de los y las estudiantes, es que el presente artículo aborda la importancia de la radio escolar digital como un medio capaz de reforzar y mejorar los aprendizajes de jóvenes en el contexto educativo chileno. La presente comunicación define qué se entiende por radio escolar, cuáles son las potencialidades que esta herramienta aporta en el marco de la era de las tecnologías, y la relación que podemos establecer entre la radio escolar digital con los planes y programas de la asignatura de Lenguaje y Comunicación, y a su vez la potencia que este insumo entrega para el desarrollo de habilidades como la creatividad, el pensamiento crítico y el aprendizaje colaborativo.

\begin{abstract}
In the context of achieving communication skills and the need to develop skills in line with the sociocultural contexts of the students, this article discusses the importance of digital school Radio as a medium able to strengthen and improve the learning process of the youth in the Chilean educational context. Thus, this communication defines what is meant by school radio, what is the potential that this tool provides under the Age of Technology, and the relationship we can established between the digital school Radio and plans and programs of the subject of language and communication, and in turn the input that delivers for the development of skills such as creativity, critical thinking and collaborative learning.
\end{abstract}

Palabras clave

Palabras clave: radio escolar digital, aprendizaje, habilidades siglo XXI

Key Key words: digital school radio, learning, XXI century skills
words

1 Este artículo es una síntesis del proyecto presentado el año 2014 a la Facultad de Comunicaciones de la Pontificia Universidad Católica de Chile para optar al grado académico de Magíster en Comunicación Social con mención en Comunicación y Educación "Radio escolar digital para estudiantes de enseñanza media del Colegio Altazor" 


\section{Introducción}

El desarrollo del lenguaje es uno de los objetivos fundamentales de la educación escolar en todos sus niveles, debido a que se constituye como la principal herramienta con la que el ser humano construye y comprende el mundo que lo rodea, a la vez que entra en diálogo consigo mismo y con otros/as. El lenguaje sustenta el pensamiento crítico y reflexivo con el que el estudiante se enfrenta a su entorno. A su vez, permite desarrollar su pensamiento y sus conocimientos, expresarlos y relacionarlos entre sí, incorporándolo a una comunidad cultural en particular.

Por otro lado la educación de hoy se plantea bajo un modelo Constructivista', que apunta a que el estudiante sea el articulador de su conocimiento con el diálogo como eje concatenador entre la instancia de aprendizaje y los participantes: profesores y alumnos a través de los que Vigotski (2010) llama Zona de Desarrollo Próximo. Con este paradigma educativo se espera que el estudiante se plantee con mayor iniciativa y dominio en la clase y comparta con el resto de sus compañeros y compañeras sus inquietudes, conocimientos y errores los que de manera conjunta consolidarán los aprendizajes necesarios para el grupo; donde el rol del docente es de facilitador del aprendizaje. Este paradigma propende a desarrollar ambientes más estimulantes, con enfoques didácticos participativos donde el diálogo, la lectura, la escritura y la investigación estén al servicio de la comunicación y la interacción de los participantes.

Bajo este marco toma relevancia la radio escolar, ya que a través de ella se trabaja el lenguaje verbal en todas sus dimensiones, sirve como canal de comunicación e información para la comunidad y vincula de una manera más participativa a los estudiantes con su establecimiento y entorno. Además, se potencian competencias comunicacionales y habilidades como la creatividad, el pensamiento crítico y el aprendizaje colaborativo en una plataforma muy atractiva para los jóvenes de hoy, sobre todo considerando que estos jóvenes -nativos digitales (García, F., Benito M., Portillo J. \& Romo J., 2008:1) - tienen una manera de relacionarse con el mundo muy distinta a la de hace una década.

La radio escolar digital, además de difundir la información relevante para los alumnos y alumnas -actividades curriculares y extra programáticas, entrevistas, noticias, entre otras-, permite que los estudiantes interactúen y transmitan sus opiniones a través de

1 Ver: Vigotsky, L. (2010). Pensamiento y Lenguaje (2ED.). Ed. Paidós Ibérica. 
programas radiales a sus compañeros y compañeras, lo que es relevante si queremos jóvenes comprometidos con la institución en la que se forman y con los asuntos de su medio sociocultural. Difundir la información a través de un canal eficaz es sumamente relevante en toda institución educativa, ya que da paso a que los actores se apropien de los lineamientos que tiene el establecimiento y estén al tanto de todas las situaciones emergentes que diariamente surgen en el contexto escolar. Por lo tanto, la difusión y la interactividad que se geste entre los estudiantes con sus propios contenidos, posibilitan la vinculación con su establecimiento de forma activa, informada y reflexiva y el desarrollo de competencias y habilidades con las que puedan enfrentar los desafíos que enfrenten cuando adultos.

Este artículo, por un lado, recoge parte de la literatura existente sobre radio escolar y sus aportes en el aula y, por otro, elabora una propuesta que relaciona las habilidades del siglo $\mathrm{XXI}$, propuestas por el sitio EducarChile y la OCDE, con la radio escolar para el Colegio Altazor de Puente Alto².

\section{La radio en el contexto de convergencia}

En la actualidad, la radio vive un proceso de transformación debido a las nuevas tecnologías con las que está interactuando la sociedad. Estas tecnologías han permitido que los usuarios hagan uso de este medio no solamente con el aparato radial común sino que puedan acceder a él a través de distintas plataformas. La digitalización de la radio y la influencia de Internet sobre su configuración permiten abrirse camino más allá de las tradicionales frecuencias AM y FM, adoptando nuevos formatos, interacción con los usuarios por nuevos dispositivos y narrativas multimedia y multiplataforma (López, 2009).

Los medios de comunicación se ven afectados en su dimensión cultural y mediática pues se insertan en un contexto de convergencia mucho más amplio en el que los contenidos y los soportes interactúan generando nuevas redes a nivel social, económico y tecnológico. Como señala Henry Jenkins (2006) "este es un proceso de la vida cotidiana, que cambia de manera intensa los hábitos de consumo de medios". Por lo tanto la radio, como medio de comunicación, ve afectada las relaciones que establece con los oyentes, las que comienzan

2 Puente Alto es una ciudad y comuna chilena, ubicada en la Región Metropolitana de Santiago. Es la capital de la Provincia Cordillera, la cual incluye además a otras dos comunas. De acuerdo al Instituto Nacional de Estadísticas, en el censo 2012 la cantidad de habitantes corresponde a 586.509.Al norte limita con la comuna de La Florida, al sur con la comuna de Pirque, al este con la comuna de San José de Maipo y al oeste con las comunas de La Pintana y San Bernardo. 
a cambiar debido a que las rutinas del oyente con el medio son distintas. Este nuevo usuario de la radio es considerado según Débora López (2009) un oyente-internauta, que busca la información en nuevas y diversas plataformas, que consume radio en nuevos aparatos y que exige de ella nuevas estrategias narrativas y de diálogo.

\section{Radio escolar como medio de aprendizaje}

Según lo que plantea Mario Kaplún "Las radios educativas o escolares son todas aquellas que procuran la transmisión de valores, la promoción humana, el desarrollo integral del hombre y de la comunidad; las que se proponen elevar el nivel de conciencia, estimular la reflexión y convertir a cada persona en agente activo de la transformación de su medio natural, económico y social 1992:32)". De acuerdo a lo anterior, la radio escolar es una herramienta valiosa que permite dinamizar la comunicación de la institución escolar, generando un espacio de participación activa e involucramiento de quienes componen la comunidad educativa con los asuntos propios de la escuela a través de la expresión. Favorece la reflexión dando paso al desarrollo del pensamiento crítico en torno a temas propios de la comunidad y de su entorno inmediato. A su vez, Juan José Perona señala: “las radios escolares permiten ampliar el conocimiento del entorno político, económico, social, cultural y natural que envuelve a los estudiantes (2001: 1)", todo ello a partir de la resignificación de los contenidos con los que el estudiante dialoga. Por otro lado, la radio escolar se define como una "experiencia educativa y escolar, entendida como herramienta pedagógica y didáctica, y como canal de expresión para producciones relacionadas con contenidos curriculares o de interés socioeducativo y comunitario" (Ministerio de Educación de la Nación Argentina, 2011,:6), definición que vincula la radio con estrategias didácticas que potencian el aprendizaje de los estudiantes en las asignaturas del currículum escolar. En esta misma línea, Jesús Jiménez Martínez (2001) sostiene que la inclusión de los medios de comunicación al aula permiten el uso de metodologías activas, participativas, abiertas, investigadoras y motivadoras que hacen de la escuela un lugar diferente.

El mismo autor destaca la conexión de los medios de comunicación en la escuela con el sector de Lenguaje y Comunicación pues los estudiantes hacen uso de estrategias o técnicas de estudio con las que refuerzan la lectura, dramatización, síntesis, creación, colaboración, personalidad, dicción, etc.; estrategias claves en la asignatura que apuntan a formar estudiantes comunicadores. 
El colombiano Diego Flores Hurtado (2009) pone más acento -a diferencia de los citados anteriormente- en la necesidad que tienen los jóvenes de participar siendo protagonistas y demostrando su liderazgo, pues las generaciones de hoy necesitan expresar lo que conocen y experimentar de manera activa y participativa, ya que, al mismo tiempo, cuestionan los sistemas tradicionales de educación, deseando explorar nuevos códigos, tecnologías y formas de hacer. En este aspecto, la radio escolar es una herramienta didáctica que posibilita la vinculación de los estudiantes con la institución, pues les da espacios de participación a través de la producción de contenidos y la interacción con el resto de sus compañeros.

Una definición que engloba varias de las definiciones anteriores es la planteada por José Guanca y Ana Sofía Pérez (2012), quienes señalan que la radio escolar, por un lado, es un instrumento de comunicación y, por otro, es un hecho cultural, en tanto que en el primero al hacer radio se mezclan códigos, formatos, normas, criterios y en el segundo, se remite a la oralidad, al espacio de identidad y al conjunto de saberes de sujetos en vinculación con el entorno. Además permite vincular áreas del currículum que aporten al proceso de enseñanza de los estudiantes. Es decir, las radios escolares potencian la vinculación de los estudiantes con su institución, la eficaz difusión de la información y el desarrollo de la expresión oral.

Alma Montoya (2007) puntualiza que la radio escolar utilizada como estrategia didáctica fortalece la creatividad de los niños y de los jóvenes pues estos se apropian del lenguaje sonoro-radial y lo utilizan para expresar sus conocimientos, emociones, inquietudes, apreciaciones e información. Además los contenidos surgen de la propia elaboración de los estudiantes quienes recrean la información. La autora pone acento en la socialización de la visión de mundo de los estudiantes, ya que se pueden comunicar a partir de la interpretación o resignificación de los contenidos que surgen en su entorno. También, al implicar el uso de la creatividad como eje fundamental en la apropiación y elaboración de contenidos, da espacio para que se genere aprendizaje significativo en un clima de autonomía cognitiva, imaginación, entretención y uso de tecnología.

En relación a las experiencias de radios escolares, éstas "comportan, en la mayor parte de los casos, beneficios directos en materia formativa para el alumnado que participa en ellas, de ahí que representen una actividad complementaria óptima en la mayor parte de las áreas en que se organiza el currículo escolar" (Blanco, E; Gómez, B \& Paniagua, F, 2007:47) 
Estos autores, en concordancia con Jesús Jiménez Martínez y el texto del Ministerio de Educación de la Nación Argentina citados anteriormente, señalan la importancia de la vinculación de la radio escolar con el currículum escolar en cualquiera de sus asignaturas, explicitando la posibilidad de éxito en la medida que se conecte con el currículo de forma complementaria.

Para Elena Blanco et. al. "los alumnos también pueden convertirse en protagonistas de la radio, utilizando el medio como canal de expresión y poniendo en práctica su creatividad comunicativa" (2007:40). La participación en un taller de radio o en una emisora escolar es la vertiente más provechosa de la experimentación con el medio sonoro, pues fomenta el trabajo en equipo, potencia la expresión oral de los alumnos y estimula su imaginación.

En cuanto a las modalidades educativas de la radio escolar, las experiencias en Españasegún señalan Juan José Perona \& Mariluz Barbeito (2007)- tienen un denominador común: se llevan a cabo fuera del horario escolar (es decir, como actividad extraescolar), aunque buena parte de los productos que conforman la programación se elaboran en horas de clase. Los autores también advierten que la radio como actividad extraescolar es una de las maneras más usuales de utilizar el medio radiofónico en los centros educativos. Por lo tanto, considerar la combinación de trabajo en horas de clases y en horas fuera de la jornada escolar son una buena posibilidad de llevar una radio escolar a la práctica dada la cantidad de horas que tiene la jornada escolar completa en nuestro país y las horas destinadas a la asignatura de Lenguaje y Comunicación según el ministerio. Además se evita la sobrecarga de tareas de los estudiantes a realizar fuera de la sala de clases.

\section{Radio escolar en Internet}

Los niños/as y jóvenes de hoy conviven diariamente con diferentes aparatos tecnológicos como computadoras, reproductores de DVD y de Mp3, celulares, consolas de videojuegos y-por supuesto- Internet (García, F., Benito M., Portillo J. \& Romo J., 2008.: 2), aparatos o medios que forman parte de su vida y que dejan a la escuela anacrónicamente distante en cuanto a las prácticas educativas enfocadas al aprendizaje de los y las estudiantes, pues se continúa educando en base a las propuestas tradicionales las que distan mucho de cómo están aprendiendo y con qué están interactuando los alumnos y alumnas en la actualidad. Es de suma relevancia incorporar prácticas metodológicas lúdicas e innovadoras que impliquen el uso de la tecnología en pos del aprendizaje. Y dada la importancia de la 
incorporación de la tecnología es que la radio escolar es vista como una oportunidad en la escuela, ya que con los avances tecnológicos de hoy es posible grabar los programas radiales y alojarlos en la web como cápsulas de audio que sean descargables para los auditores. "La utilización de la radio por Internet y la implementación de su dinámica se organizan en un auténtico sitio web radiofónico, con el que los estudiantes, profesores, directivos y la comunidad educativa en general puedan interactuar, involucrarse y además sentirse identificados" (Szyszko, Neri, \& Cataldi, 2010). Además, con el empleo de Internet, resulta posible ofrecer una dimensión sonora a los mensajes que van a recibir los radioescuchas pues la tecnología disponible permite la creación de un prototipo de radio escolar accesible a través de una página web, sin las dificultades económicas y legales que lleva aparejada una radio analógica. Por otra parte, al estar alojada en Internet permite que el usuario descargue a su computador u otro ordenador los programas cuando quiera y los escuche en cualquier momento.

La aparición de la tecnología streaming ${ }^{3}$, difusión de audio o video por Internet, ha posibilitado la compresión de formatos de información sonora en tiempo real para después transmitirla. De esta manera el receptor recibirá la emisión si posee el reproductor correspondiente para descodificar el contenido (Pestano Rodríguez y Gabino Campos, 2006). Las posibilidades que brinda la tecnología permiten incorporarla en el sector educativo como apoyo de diversas habilidades entre ellas la creatividad. Emma Rodero (2004) señala

\footnotetext{
"La tecnología se coloca entonces al servicio de la creatividad porque sus propias condiciones y características obligan a una revisión de los quehaceres radiofónicos. Además, no debemos olvidar que la nueva tecnología ha nacido ya con estos jóvenes y, por tanto, en la misma dinámica y hábitos de consumo que ellos mismos. Por acercarnos a una realidad más palpable que la radio digital, observamos cómo las propias características de la red se aproximan a los hábitos de consumo de los jóvenes y, por ello, se deduce que Internet puede convertirse en un medio idóneo para satisfacer sus necesidades y fomentar el empleo radiofónico”. (p. 141)
}

\footnotetext{
3 Consiste en una tecnología utilizada para permitir la visualización y la audición de un archivo mientras se está descargando, a través de la construcción de un buffer por parte del cliente, una vez que este se ha conectado al servidor, el buffer del cliente se va llenando de la información descargada y se va reproduciendo en el ordenador. El sistema se encuentra sincronizado, tal que, una vez terminada la reproducción del contenido del archivo, finaliza la descarga (siempre y cuando no existan interrupciones en el envío del archivo). En http://www.mastermagazine.info/termino/6781.php\#ixzz326FkGjTE
} 


\section{La radio escolar digital y su relación con los planes y programas de Lenguaje y Comunicación}

La propuesta formativa del Marco Curricular y Actualización lo a IVo Medio, en el sector de aprendizaje Lenguaje y Comunicación apunta hacia el desarrollo de las competencias comunicativas que los estudiantes requieren para su formación integral, dado que el lenguaje "es la base de las demás áreas del saber y constituye en sí mismo una herramienta fundamental para la integración de las personas en una sociedad moderna, participativa y democrática, se propicia el desarrollo del pensamiento crítico y reflexivo, la creatividad y el diálogo" (Ministerio de Educación, 2009 :31). Para llevar a cabo lo anterior es necesario enriquecer el lenguaje con el que los estudiantes ingresan al sistema, ampliando y mejorando la comunicación oral y el acceso al lenguaje escrito a través de la lectura y la escritura, por lo tanto se debe potenciar la expresión y la comunicación de opiniones, ideas, sentimientos y convicciones propias, con claridad y eficacia, pues a través de este proceso se estimula "una actitud que apunta a respetar y valorar las ideas y creencias distintas de las propias, a reconocer el diálogo como fuente permanente de humanización, de superación de diferencias y de aproximación a variados conocimientos" (Op.Cit). En este contexto, la radio es un medio que posibilita el desarrollo de la propuesta de la asignatura de Lenguaje y Comunicación, ya que como medio de comunicación no se concibe si no es en base a la comunicación como pilar fundamental, lo que da espacio para que los estudiantes se formen como comunicadores eficaces en diversas situaciones comunicativas.

Lo anterior se detalla con más profundidad en la descripción del eje de comunicación oral donde los planes y programas definen el eje como

\footnotetext{
..."es un punto de partida para desarrollar todos los otros ámbitos de la competencia comunicativa, ya que, a partir de esta se adquieren las habilidades propias de la lectura y la escritura. En general, las personas desarrollan esta habilidad de manera natural durante su vida y en las distintas instancias del vivir cotidiano. Sin embargo, las interacciones informales no proveen las herramientas necesarias para participar activamente en todos los ámbitos de la sociedad. La educación media es el lugar en que se refuerzan las habilidades adquiridas en los ciclos básicos, es decir, respetar los turnos de habla y escuchar con atención, adecuarse a la situación comunicativa, utilizar un vocabulario preciso, expresarse con claridad y fundamentar sus opiniones. A esto se suma ejercitar de manera sistemática la exposición formal y el debate, que involucran habilidades fundamentales para alcanzar el éxito en la educación superior y en el mundo laboral (Ministerio de Educación, 2009.:7).
} 
Por lo tanto, la radio escolar digital es una herramienta que está al servicio del desarrollo de competencias comunicativas en los estudiantes, ya que les permite participar activamente en la creación de contenidos asociados con su vida escolar y aprender a escucharse atentamente, respetar sus turnos de habla, tomar decisiones, etc.; todas ellas fundamentales para vivir en sociedad.

La propuesta curricular ministerial -base en la planificación de la asignatura en el Colegio Altazor - incorpora actividades que buscan la interacción y la discusión constante dentro del aula, considerando los distintos tipos de lenguaje que se dan en todo diálogo y la creación de contenidos a nivel individual y colaborativo. Tanto los aprendizajes esperados como los contenidos mínimos del eje se pueden trabajar a través de la radio pues esta permite la interacción de los estudiantes a través de discursos de distinta intención, distintas modalidades discursivas y distintas situaciones comunicativas en formatos como foro, debate, entrevista, etc. La radio es una herramienta pedagógica con la que se puede potenciar el eje de comunicación oral de los estudiantes, entre otros beneficios, pues posibilita el desarrollo de habilidades de comunicación y las competencias necesarias para desempeñarse como eficientes receptores y productores de distintos tipos de discursos en variadas situaciones comunicativas.

\section{La radio escolar y su relación con las habilidades para el proceso de enseñanza aprendizaje en la era de las tecnologías de la información}

En el contexto de las nuevas tecnologías con las que los jóvenes están interactuando, la educación ha tenido que cambiar del paradigma de los contenidos al paradigma de las habilidades y las competencias, que apuntan principalmente a que el estudiante pueda aplicar lo aprendido al contexto en el que se sitúa. El uso de redes sociales, herramientas computacionales y formatos de aprendizaje diferentes e informales como cómics, videojuegos y foros virtuales nos advierte la existencia de otras maneras de aprender de los estudiantes, donde el acceso a la información y a la interacción con el mundo virtual y tecnológico es amplio e ilimitado. Los jóvenes de hoy se caracterizan por relacionarse horizontalmente con quien deseen, manifestar sin miedos sus opiniones y conocerse por sus avatares y nicknames. Son una generación empoderada tecnológicamente, conectada a través de diversas plataformas virtuales y que disfruta de sus tiempos libres con videojuegos y chat (Paulsen, 2011). 
Este nuevo paradigma necesita de un profesor que medie el aprendizaje de sus estudiantes, a través de la autonomía y la aplicación de lo aprendido a la realidad de los estudiantes, con el fin de enseñarles a pensar y resolver desafíos. Estas habilidades, de acuerdo a lo que señala el artículo Habilidades para el siglo XXI de la plataforma educativa EDUCARCHILE (2013), serían: colaboración, creatividad y pensamiento crítico. "El desarrollo de estas habilidades debe ser parte íntegra de la planificación de las clases y evaluaciones, lo que puede hacerse por medio de instrumentos de evaluación sobre el trabajo en el aula y el desempeño de los estudiantes". Por otro lado, el Instituto de Tecnologías Educativas (2010) en su artículo Habilidades y competencias del siglo XXI para los aprendices del nuevo milenio en países de la OCDE, señala que "estas habilidades y competencias se denominan normalmente habilidades y competencias del siglo XXI con el fin de indicar que están más relacionadas con las necesidades de los modelos emergentes de desarrollo económico y social que con aquellas del siglo pasado al servicio del modo industrial de producción (ITE, 2010)". Por lo tanto estas habilidades desempeñan una labor fundamental: permitir el andamiaje entre avances tecnológicos y crecimiento/evolución humana, pues como ya se ha mencionado antes, la mera utilización del aparato tecnológico no es suficiente, se requiere del aprendizaje de una nueva manera de vivir, de relacionarse, de desplazarse y convivir. Unido al raciocinio, diálogo y creatividad entre sus involucrados.

Dado lo anterior la radio escolar es la herramienta pedagógica perfecta para trabajar estas tres habilidades del siglo XXI: aprendizaje colaborativo, creatividad y pensamiento crítico.

\section{El aprendizaje colaborativo}

En metodologías que implican el aprendizaje colaborativo, lo que se busca es que exista integración dentro de los grupos de trabajo. De este modo, a diferencia del trabajo cooperativo, donde las labores se distribuyen y cada integrante se aboca a desarrollar una labor determinada para luego unir los esfuerzos, en esta dinámica las tareas y supervisiones son realizadas por todos los miembros. Es decir, no existe la figura de un coordinador, sino que cada uno puede actuar como ejecutor y revisor de lo que se está haciendo (Carrió, 2007; Moranchel, Vázquez, Herranz, 2011).

Se vincula con la radio escolar debido a que la radio necesita de un trabajo integrador en el que cada participante ejecute y revise lo que se está haciendo, pues para sacar al aire un programa requiere que todos los involucrados aporten con las tareas que les han sido 
asignadas como equipo lo que permite desarrollar habilidades personales y sociales. Por otro lado se cumplen los cinco supuestos planteados por Barkley, Cross y Howell Major (2007):

- Intencionalidad: los estudiantes tendrán claridad respecto a cuál es el objetivo principal que tienen como equipo, el que se relaciona específicamente con sacar al aire un programa determinado.

- Trascendencia: las actividades diseñadas para poner al aire los programas tendrán directa relación con contenidos curriculares de la asignatura de lenguaje y comunicación las que serán evaluadas como actividades de aula.

- Reciprocidad: cada integrante del equipo tendrá tareas asignadas que involucran el resultado final de la actividad, por lo tanto es vital la participación de todos para conseguir la meta planteada.

- Significado: los estudiantes debe conocer la importancia que tiene para la comunidad educativa y para ellos mismos su participación en un proyecto de tal envergadura, además es fundamental la motivación que manifiesten en la organización y la generación de contenidos donde son ellos los protagonistas y los contenidos se relacionan con su quehacer escolar.

- Autovaloración: cada participante podrá observar los avances que va teniendo en ámbitos como comunicación oral o participación de la vida escolar de manera más protagónica, por lo tanto podrá verse a sí mismo como un comunicador radial.

Todo lo anterior es posible llevarlo a cabo en la medida que exista un docente que cumpla el rol de mediador de las actividades y del aprendizaje otorgándole al alumno autonomía y autorregulación de las prácticas específicas como integrante del proyecto de radio escolar. La resignificación de los contenidos, la participación activa y la motivación por el aprendizaje enlazado con su contexto son pilares fundamentales para desarrollar esta habilidad.

\section{La creatividad}

En un siglo donde la humanidad se ve enfrentada al estrés, fragmentación, consumismo, soledad, diferencias sociales y calentamiento global es lógico imaginar las situaciones diversas con las que convive, soluciona, genera, etc. En este contexto, la creatividad según 
Olena Klimenko (2008) "adquiere significado como un valor cultural que permite generar soluciones eficaces para las problemáticas contemporáneas y como una necesidad fundamental del ser humano, cuya satisfacción permite alcanzar una mayor calidad de vida." Lo anterior da cuenta de la necesidad de formar personas que sepan, a través de la creatividad, enfrentarse al mundo y actuar en él con herramientas atingentes a la velocidad con la que el mundo avanza y donde será necesario adaptarse y crecer. Por otro lado, en este nuevo escenario, bien podría estimularse este proceso creativo a través de la adaptación de espacios favorables y estimulantes para las personas con el fin de desarrollar estrategias o productos creativos a compartir con el entorno social en el que se insertan los individuos (González, 1994; Martínez, 1998; Mitjan, 1997; Betancourt, 2007; Chibas, 2001). Por lo tanto, la creatividad es una habilidad que al ser estimulada y trabajada se puede desarrollar y potenciar aplicada a situaciones cotidianas en la que los individuos la puedan poner en acción, pues de acuerdo a lo que señala Klimenko (2008) "la capacidad creativa de los individuos no está determinada al nacimiento, sino que se desarrolla a través de los años gracias al estímulo constante en un espacio de aprendizaje dispuesto a dar protagonismo a esta habilidad."

Es una habilidad que, sin duda, se relaciona con la radio escolar ya que permite que los estudiantes dejen llevar su imaginación a la hora de crear los contenidos propios de la radio: géneros, programas, publicidad, entre otros.

La radio permite que se estimule esta habilidad a través del trabajo aplicado a la cotidianidad de los estudiantes, donde ellos puedan poner en acción su aprendizaje. Lo que da pie para que a través de la indagación, la entrevista, el análisis de los contenidos o noticias locales, gustos y lecturas se promueva el aprendizaje significativo y reflexivo, permitiendo a su vez que la mente del alumno y alumna describa y transforme su entorno a través de las herramientas de aprendizaje de las cuales dispone.

En el siglo XXI, los espacios educativos deben propiciar un clima en donde la creatividad de los estudiantes se vea constantemente desafiada, por lo tanto, la radio es un espacio donde el diálogo, el consenso y la adecuación del material los invitan a replantear la información con la que conviven. Por otro lado, la participación de los estudiantes en la radio les permite relacionarse con tecnologías con las que ellos tienen mayor cercanía y los motiva a la experimentación con los recursos. Los conecta con su entorno desde una perspectiva más integradora, participativa y propositiva. Actividades que involucren un rol 
protagónico del estudiante como constructor de conocimiento de manera colaborativa significarán un avance en el camino de la formación de jóvenes más preparados para enfrentar el siglo en el que les corresponde vivir.

\section{El pensamiento crítico}

Dewey (1982) define pensamiento crítico como "una consideración activa, persistente, cuidadosa de una creencia o forma supuesta del conocimiento a la luz de los fundamentos que lo sustentan y a las conclusiones a las que se dirige". Matthew Lipman (1998) define pensamiento crítico como el pensamiento responsable que facilita el juicio porque se basa en criterios, es autocorrectivo y sensible al contexto. El autor pone énfasis en la emisión de juicios sustentados en criterios válidos que permitan apoyar las opiniones que comparten con su entorno diariamente. Tanto Dewey (1982) como Lipman (1998) distinguen el contexto como un espacio en el que se produce la construcción de pensamiento reflexivo o crítico, entendiendo que las experiencias cotidianas a las que se enfrentan los individuos les permitirán establecer juicios, resolver conflictos o tomar decisiones en un momento específico. El contexto determina el pensamiento crítico en la medida de que no se puede desprender al individuo del tema o las circunstancias en que se suscriben sus razonamientos o la manifestación de las ideas.

La radio escolar es un medio en el cual se puede desarrollar esta habilidad debido a que, a través de ella, los estudiantes podrán manifestar sus reflexiones y perspectivas del mundo en un espacio que les permita generar debates, foros o mesas redondas en las que se discutan temas de relevancia para ellos y/o de contingencia nacional. Los alumnos y alumnas tienen mucho que decir y aportar a su comunidad educativa, pero para hacerlo necesitan de los espacios adecuados que canalicen sus apreciaciones del mundo contemporáneo. Dado lo anterior, la radio escolar puede apuntar al desarrollo de esta habilidad en la medida que se integren espacios donde los estudiantes opinen reflexivamente sobre contenidos que pueden ir desde los gustos en la comprensión lectora, gustos musicales, discusiones sobre temas de actualidad, debates de las listas que se presentan para centros de alumnos o problemáticas del colegio, etc., vistas desde la perspectiva del estudiante, entre muchos otros programas que potencien el pensamiento crítico. Trabajar colaborativamente implicará necesariamente que se enfrenten a situaciones donde tengan que tomar decisiones y resolver una serie de conflictos propios de toda actividad o proyecto, por lo tanto los estudiantes se pueden enfrentar a diversas situaciones donde exponer sus puntos de vista y proponer soluciones les permitirá avanzar y crecer. 


\section{Reflexiones finales}

Considerando los procesos de cambio que está viviendo la educación en Chile, se vuelve sumamente importante plantear propuestas que impliquen prácticas metodológicas lúdicas, cercanas a los jóvenes y relacionadas con la tecnología que, como se ha visto, avanza rápidamente entre los y las estudiantes que los docentes educan en la actualidad. La radio es un medio de comunicación que presenta características atractivas y trascendentales para los educando -y para la ciudadanía en general-como la participación, interactividad, inmediatez, portabilidad, por señalar algunas; que hacen de este medio un recurso fresco e innovador de incorporar en el aula.

Hacerse cargo de las competencias que se deben desarrollar en los estudiantes con el fin de que estos pueden insertarse a los diversos espacios que elijan en el futuro con herramientas sólidas y atingentes a los múltiples escenarios que deberán enfrentar, ya sea como actores protagónicos o secundarios. La creatividad y la resolución de problemas son esenciales a la hora de trabajar colaborativamente, por lo tanto se debe preparar a los jóvenes a enfrentarse a esta nueva forma de vivir en la sociedad del siglo 21.

En la sala de clases los y las estudiantes disfrutan mucho más el aprendizaje cuando éste les propone actividades que involucren herramientas tecnológicas y participación, más que exposición exclusiva del profesor, debido, principalmente, a la necesidad y la capacidad que tienen los alumnos y alumnas de atender a varias cosas a la vez. Lo anterior implica que se busquen nuevas alternativas, que no solo sean innovadoras sino que además formen en habilidades acorde a los tiempos.

La incorporación de una radio escolar digital en un establecimiento educacional sin duda significará un cambio positivo y fundamental en el desarrollo de habilidades y competencias que cada asignatura del currículum tiene por objetivo. Lo importante ahora es entregar estas herramientas a los docentes y prepararlos para enfrentar nuevos desafíos que motiven a sus estudiantes a aprender. Los resultados que estas iniciativas aporten a mejorar la calidad de la educación que se entrega en los colegios dejan un nicho de estudio interesante de abordar en otras investigaciones, que busquen cuantificar los resultados que estas nuevas prácticas traen consigo y que analicen la efectividad de las herramientas pedagógicas que están utilizando los docentes para formar a los jóvenes del futuro. 
Referencias Balsebre, A., (1994). El lenguaje radiofónico, Madrid:Cátedra.

Barkley, E. F., Cross, K. P. y Major, C. H. (2005). Collaborative learning techniques: A hand-book for college faculty. San Francisco: Jossey-Bass Publishers.

Beltrán, J., y Pérez, L. (1996): "Inteligencia, pensamiento crítico y pensamiento creativo", en Beltrán, J.y Genovard, C. (Eds.): Psicología de la instrucción I. Variables y procesos. (pp. 429-503), Madrid : Síntesis.

Blanco, E; Gómez, B \& Paniagua, F. (2007). La utilización de la radio como herramienta didáctica. FISECEstrategias. Año III (6). 35-50. [En línea]. Recuperado de: http://www.cienciared.com.ar/ra/usr/9/369/ n6_m3pp35_50.pdf

Bruning, R. H., Schraw, G. J. \& Ronning, R. R. (1999): Cognitive psychology and instruction. Englewood Cliffs, N. J., Prentice Hall. (Trad. C ast.: Psicología cognitiva e instrucción. Madrid:Alianza, 2002).

Cebrián, M., (2011) "La radio en el entorno de las multiplataformas de comunicaciones", en RadioLeituras, num. 02, año II, [En línea] Recuperado de: http://radioleituras.wordpress.com

Collazos, C.; Guerrero, L. \& Vergara, A. (2001). Aprendizaje Colaborativo: un cambio en el rol del profesor. Trabajo presentado en Tercer Congreso de Educación Superior en Computación, Punta Arenas.

Contreras, D. (2008). CIVIT@S.NET: Alfabetización digital para ciudadanos en red. En MINEDUC (Coord.), Experiencias de Innovación en Informática Educativa 2007 (pp. 401-432). Santiago de Chile: MINEDUC.

Dewey, J. (1982). Como pensamos. Nueva exposición de la relación entre pensamiento reflexivo y proceso educativo. Barcelona: Editorial Paidós.

Dido, J. y Barberis S. (2008) La radio en la escuela.Un recurso didáctico de gran valor educativo.. . Buenos Aires: Universidad Nacional de La Matanza.

EducarChile. Habilidades del siglo XXI. En portal educativo EDUCARCHILE [En línea]. Recuperado de: http://www.educarchile.cl/ech/pro/app/detalle?!D=219621

Elstein, S. (1999). Nuevas tecnologías y educación. Hacia una nueva perspectiva en la formación de profesores. Nuevas tecnologías, nuevos entornos sociales y culturales. Contextos de educación, IV [en línea] Recuperado de: http://www.unrc.edu.ar/publicar/cde/Elstein.htm.

Flores, D. (2009). La emisora escolar: herramienta pedagógica en el proceso de enseñanza aprendizaje de los valores y en la transversalización temática de las áreas. Instituto Técnico de Santander de Quilichao.

García, F., Benito M., Portillo J. \& Romo J. (2008). Nativos Digitales y modelos de aprendizaje, [en línea] Recuperado de: http://spdece07.ehu.es/actas/Garcia.pdf

Guajardo, V. \& Ortega, R. (2008). TIC y aprendizaje. Servicio de la comunidad rural. En MINEDUC (Coord.), Experiencias de Innovación en Informática Educativa 2007 (pp. 207-232). Santiago de Chile: MINEDUC.

Guanca, J. \& Pérez, A. (2012). La radio como herramienta de enseñanza aprendizaje. Exposición de dos casos al interior de Tucumán. XIV Congreso REDCOM, Investigación y extensión en comunicación: sujetos, políticas y contextos. 
Herrera, S. (2003). Rasgos diferenciales de la radio como medio de participación. Revista de Comunicación, 25-40.

Instituto de Tecnologías Educativas (2010), Habilidades y competencias del siglo XXI para los aprendices del nuevo milenio en países de la OCDE. España.

Jenkins, H. (2008). La cultura de la convergencia de los medios de comunicación. Nueva York:Editorial Paidós.

Jiménez, J. (2001). La radio a la escuela. Contextos educativos (4), 297-313.

Kaplún, Mario. (1992). A la educación por la comunicación. La práctica de la comunicación educativa. Santiago de Chile: UNESCO.

Klimenko, O. (2008). La creatividad como un desafío para la educación del siglo XXI. Educación y Educadores, 191-210, [en línea] Recuperado de: http://www.redalyc.org/pdf/834/83411213.pdf

Lipman, M. (1998). El pensamiento crítico: ¿Qué puede ser? En: Itinerario pedagógico. Bogotá. 28 -30, 206.

Litwin, E. (1995). Tecnología Educativa. Políticas, historias, propuestas. Buenos Aires: Paidós.

López, D. (2009). Radiojornalismo hipermidiático: onvergenc e perspectivas do jornalismo de rádio all news brasileiro em um contexto de onvergencia tecnológica. 2009. 301 f. (Tesis de Doctorado en Comunicación y Cultura Contemporáneas - Universidad e Federal da Bahia (UFBA), Salvador.)

Marchesi, A., Martin, E., Casas, E., Ibáñez, A., Monguillot, I. \& Riviere, V. (2003). Tecnología y aprendizaje. Investigación sobre el impacto del ordenador en el aula. Madrid: SM.

Martín-Laborda, R. (2005). Las nuevas tecnologías en la educación. Cuadernos / Sociedad de la información, p.5.

Merayo, A. (2007). La radio en Iberoamérica: Evolución, diagnóstico y prospectiva. España: Comunicación Social: Ediciones y publicaciones.

Merayo, A., (1999) "Tecnología e información en la radio española de fin de siglo", En: ZER Revista de Estudios de Comunicación, n¹. Bilbao: UPV/EHU, pp. 93-108. Disponible en http://www.ehu.eus/zer/ es/hemeroteca/articulo/Tecnologa-e-informacin-en-la-radio-espanola-de-fin-de-siglo/7, consultado el 27/11/15.

Merayo, A., (2000) Para entender la radio, Salamanca, Publicaciones Universidad Pontificia de Salamanca. (2a edición)

Ministerio de Educación de la Nación. (2011). Programa Nacional de Extensión Educativa "Abrir la Escuela". Buenos Aires.

Ministerio de Educación (2009). Marco Curricular y Actualización Io a IVo Medio. Santiago.

Ministerio de Educación (2012). Guías didácticas de comunicación oral: Lenguaje y Comunicación. P. 89 - 109, Santiago.

Montoya, A., \& Nadalich, I. (2007). Radio Escolar, generadora de procesos comunicativos y pedagógicos. Comunicación y escuela nº 2, pp. 17-27. 
Ortega, E. \& Guell, V. (2006). Las nuevas tecnologías: ¿Un salto al futuro? Santiago de Chile: Programa de las Naciones Unidas para el Desarrollo (PNUD).

Paulsen, F. (2011). Mucha opinión pocas ideas. En encuesta Jóvenes, participación y consumo de medios. Universidad Diego Portales. Santiago.

Perona, J. J. (2006). El Lenguaje radiofónico: Introducción. Publiradio, 2-5.

Perona, J., \& Barbeito, M. (2007). Modalidades educativas de la radio en la era digital. Revista de Comunicación y nuevas tecnologías nº 9, pp. 16-36.

Perona, J.J. (2001). "Radio escolar en Internet: un proyecto pedagógico para la Era digital". En Red Digital. Revista de Tecnologías de la Información y Comunicación Educativas. CNICE. Núm. 1. [En línea] Recuperado de: http://reddigital.cnice.mec.es/1/

RELPE (2011). Proyecto Radio Escolar Online [En línea]. Recuperado de: http://www.relpe.org/ ultimasnoticias/proyecto-radio-escolar-online/

Rodero, E. (2004). Recuperar la creatividad radiofónica: razones para apostar por la radio de ficción. Facultad de Comunicación, Universidad Pontificia de Salamanca. Análisis, 32 133-146

Szyszko, N., Neri, C., \& Cataldi, Z. (2010). La radio en la escuela media como agente participativo. Universidad Tecnológica Nacional, Facultad Regional. Buenos Aires. [En línea] Recuperado de: http:// www.quadernsdigitals.net/datos_web/hemeroteca/r_1/nr_808/a_10906/10906.html 